\title{
Pheochromocytoma Presenting as the Alternation of Hypertension and Hypotension
}

\author{
Weiqun $\mathrm{Zhao}^{1}$ and Liqun zhao ${ }^{2 *}$ \\ ${ }^{1}$ Department of B-Ultrasound, Wuxi Third People's Hospital, China \\ ${ }^{2}$ Department of Cardiology, Shanghai General Hospital, China
}

*Corresponding author: Liqun Zhao, Department of Cardiology, Shanghai General Hospital, Shanghai, China

\begin{abstract}
ARTICLE INFO
Received: 幽 February 14, 2020

Published: 幽 February 24, 2020

Citation: Weiqun Zhao, Liqun zhao.

\section{ABSTRACT}

We reported a rare case of pheochromocytoma presenting as the alternation of hypertension and hypotension, which highlighted the rare event of an extreme and cyclical blood pressure pattern in a pheochromocytoma hypertensive crisis. Early recognition is critical for selection of the appropriate treatment to terminate the effects of catecholamine storm, which prepares for surgical resection of the tumor.
\end{abstract} Pheochromocytoma Presenting as the Alternation of Hypertension and Hypotension. Biomed J Sci \& Tech Res 25(5)-2020. BJSTR. MS.ID.004274.
Keywords: Pheochromocytoma; Hypertension; Hypotension

\section{Introduction}

A 50-year-old woman presented to the emergency department and complained of chest tightness, palpitation and frequent episodes of nausea, vomiting, and headache for the past 2 hours. She had a 1-year history of type 2 diabetes mellitus and was treated with diet therapy (1500 kcal/day) and oral hypoglycemic agents' metformin (250 mg/ twice/ day). Subsequently she had fair glycemic control with a mean glycated hemoglobin (HbA1c) of $6.0 \%$ (reference range: 4.6-6.2\%). She had never had a history of hypertension and any other notable conditions. On physical examination, she was pale, in distress, and sweating profusely; Her blood pressure (BP) was 300/180 mmHg; pulse, 180 beats/ min; respiratory rate, 38 breaths/min; and oxyhemoglobin saturation, 94\% while inhaling 100\% oxygen concentration with face mask; Lung auscultation revealed clear breath sounds without crepitations; the abdomen was soft on palpation and no unusual mass was detected. Electrocardiography showed sinus tachycardia, 168 beats/min; chest X- ray was normal. Her systolic BP abruptly dropped to $60 \mathrm{mmHg}$ before phentolamine was administered. She was immediately treated with fluid expansion and dopamine hydrochloride (12 $\mu \mathrm{g} / \mathrm{kg} / \mathrm{min})$ to maintain BP levels between 70/40 mm Hg and 90/50 mm Hg. After 5 minutes BP rose sharply to $210 / 150 \mathrm{mmHg}$ and complained of chest pain, palpitation and headache. Intravenous phentolamine $(5 \mathrm{mg}$ bolus, followed by continuous infusion) was administered promptly to eradicate blood pressure. $\beta$-blocker propmnolol (10 mg/three times/day) was initiated to control tachycardia. Her systolic blood pressure dropped to $50 \mathrm{mmHg}$ again.

Her systolic blood pressure would oscillate between $60 \mathrm{mmHg}$ and $350 \mathrm{mmHg}$ at 3-5 minutes' intervals. The episode occurred unpredictably and repetitively (Figure 1). PICCO was established to monitor continuous intra-arterial pressure, which revealed unusual rhythmic alternation of hypertension and hypotension. This cyclic hemodynamic crisis continued for the next 6 hours. Hypertensive episodes were accompanied by ST-segment depression on electrocardiography, which returned to baseline during hypotensive phases. Echocardiogram showed normal left ventricular size and function (Dd/Ds $50 \mathrm{~mm} / 38 \mathrm{~mm}$, left ventricular ejection fraction $60 \%$ ) and no valvar abnormalities or asynergy and a little pericardial effusion. Abodminal ultrasound examination showed the upper pole of right adrenal gland could be detected heterogeneous mass (5.0 $\times 3.3 \mathrm{~cm}$ ) with a clear boundary. Color doppler ultrasound showed rich color blood flow was seen in parenchymal portion of the mass. Abodminal Computed Tomographic (CT) scan with contrast agents revealed a well-defined, heterogeneous mass in the right 
adrenal gland $(5.2 \times 3.4 \mathrm{~cm})$ (Figure 2$)$. The urinary echolamines on discomfort were elevated: epinephrine $68.7 \mathrm{ug} / 24 \mathrm{~h}$ (reference range, 0 - $22 \mathrm{ug} / 24 \mathrm{~h}$ ), norepinephrine $117.40 \mathrm{ug} / 24 \mathrm{~h}$ (reference range, 7- $65 \mathrm{ug} / 24 \mathrm{~h}$ ), dopamine $102.70 \mathrm{ug} / 24 \mathrm{~h}$ (reference range, 75 - $440 \mathrm{ug} / 24 \mathrm{~h}$ ). In the light of clinical symptom, abodminal CT and Laboratory data, pheochromocytoma was considered as a possible diagnosis. The patient spent 7 days in intensive cardiac care unit and experienced no further attacks. Seven days later, he underwent successful open right adrenalectomy. Pathologic study of the tissue confirmed low malignant pheochromocytoma without necrosis. After surgery the patient remains normotensive, and only continues to take oral hypoglycemic agents.

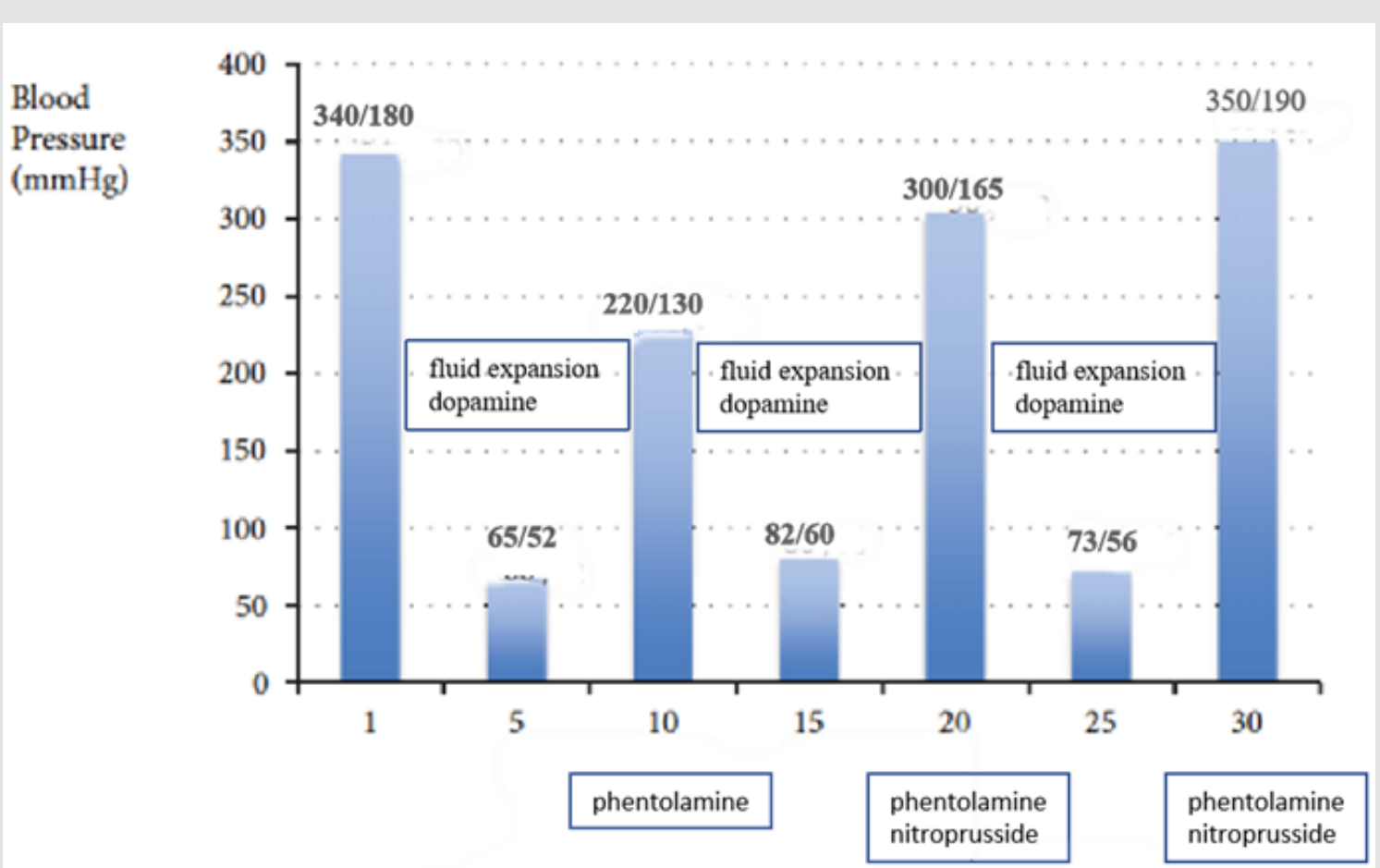

Figure 1: Blood pressure in the department of emergency.

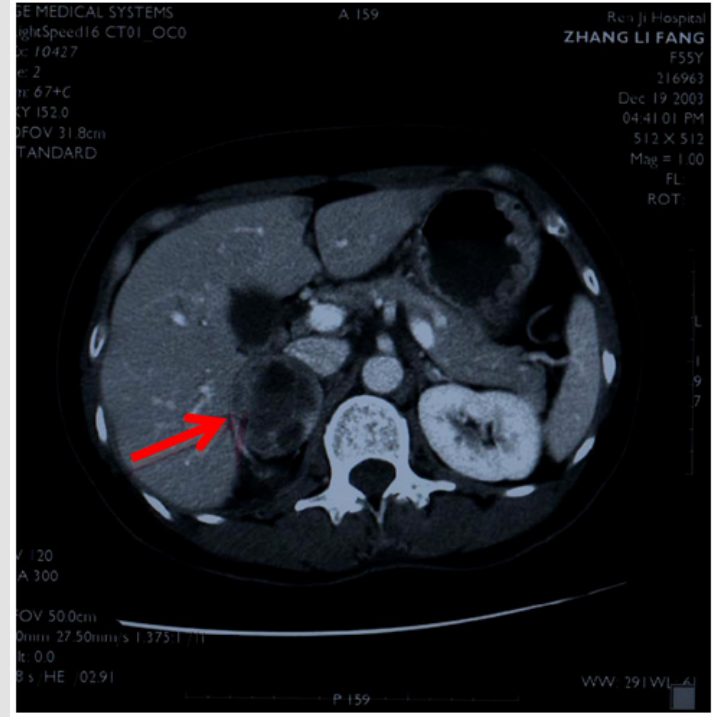

A

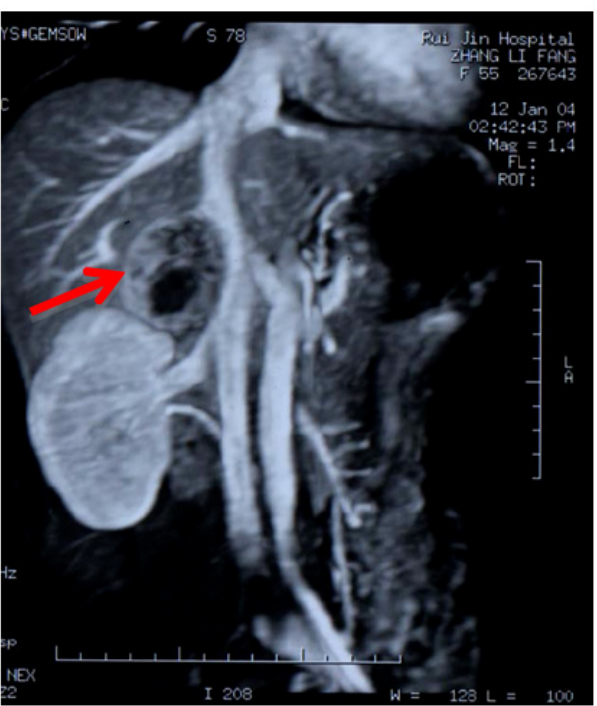

B

Figure 2: Computed tomography scan with the abdomen. A: Computed tomography scan with contrast of the abdomen. The arrow points toward the right heterogeneous adrenal mass, which measures $5.2 \times 3.4 \mathrm{~cm}$. The left adrenal gland is normal. B: The view of the abdomen on computed tomography venography shows a well-defined, heterogeneous mass in the right adrenal gland $(5.2 \times 3.4 \mathrm{~cm})$. 


\section{Discussion}

Hypertensionisthemostfrequentfindingin pheochromocytoma. More than half of the patients present sustained hypertension, whereas about $45 \%$ have Paroxysmal BP elevations during a crisis. Hypotension (especially orthostatic) may be seen in patients with pheochromocytoma. However, the alternation of hypertension and hypotension in pheochromocytoma is a rare finding. Hypertension of phaeochromocytoma is the result of catecholamines on the cardiovascular system probably coupled with enhanced sympathetic nervous system activity. Pathophysiological factors of hypotension include hypovolemia, abrupt cessation of catecholamine secretion owing to hemorrhagic necrosis in the tumor or desensitisation of adrenergic receptors [1]. Prolonged exposure to high levels of epinephrine and norepinephrine could result in arterial and venous vasoconstriction, leading to vessel wall ischemia, anoxia and enhanced permeability, which contributes to a reduction in blood volum. Abrupt cessation of catecholamine secretion and desensitisation of adrenergic receptors cause a subsequent decreasing peripheral vascular resistance. Forthmore, peripheral vascular resistance during hypertensive crisis intensively increase, leading to decreased cardiac output [2], which results in a reduction in BP. Hypotension can also be caused by a complicating cardiovascular emergency, such as heart failure due to toxic cardiomyopathy, myocardial infarction, cardiac arrhythmias, or a dissecting aortic aneurysm [3,4]. A reduction in blood volume and cardiac output would, in turn, stimulate excessive reflex catecholamine release, causing paroxysmal hypertension $[5,6]$. The increase in BP would stimulate baroreceptors in the blood vessel walls and activate a negative feedback loop, mediated by sympathetic and parasympathetic nervous systems, which would respond by decreasing peripheral vascular resistance and lowering cardiac output, causing a subsequent reduction in BP. Baroreceptors are tonically active and can respond quickly to changes in BP, which could explain the rapid alternation between hypertension and hypotension seen in this patient [7].

In gernal, pheochromocytoma is treated with a-blockers, usually combined with a b-blocker, prior to tumor removal decreases mortality associated with surgery [8]. Treatment with an $\alpha$-adrenergic receptor blocker should precede $\beta$-blockade for the treatment of Tachycardia to prevent a paradoxical increase in BP that can occur when $\alpha$-adrenergic receptors are stimulated, and vasodilating $\beta$-adrenergic receptors are blocked. Volume expansion and administration of vasoactive substances, such as dopamine and norepinephrine are needed to maintain BP at normal levels in patients presenting with shock.

The case presented here was an unusual pheochromocytoma: cyclic waves of severe hypertension alternating with hypotension.
This patient mainly secreted norepinephrine along with amounts of epinephrine but did not produce dopamine and/or levodopa, which led to hypertension. So, we choose phentolamine to control BP. In this case, hypertension alternating with hypotension is not associated with abrupt cessation of catecholamine secretion bacause this patient's pathologic tissue was low malignant Pheochromocytoma without necrosis. The cause of hypotension is due to hypovolemia or desensitisation of adrenergic receptors. Her BP rose sharply to hypertensive crisis owing to stimulating baroreceptors in the blood vessel walls and activating a negative feedback loop. Intravenous phentolamine (5 mg bolus, followed by continuous infusion) was administered promptly and $\beta$-blocker propmnolol $(10 \mathrm{mg} /$ three times/day) was initiated after phentolamine. When SBP dropped to below $90 \mathrm{mmHg}$, it is more helpful for this patient to maintain SBP between $90 \mathrm{~mm} \mathrm{Hg}$ and $100 \mathrm{~mm} \mathrm{Hg}$ by the treatment with fluid expansion and dopamine hydrochloride. The dose of intravenous medications was regulated monentarily by continuous intra-arterial pressure monitor. I got some benifit from this case. Typical pheochromocytomas mainly secrete norepinephrine along with smaller amounts of epinephrine but, in rare cases, can also produce dopamine and/or levodopa. So, we should first choose fluid expansion and dopamine to maintain BP, especially in patients with Pheochromocytoma without necrosis. BP might rise sharply to hypertensive crisis if treated with norepinephrine or epinephrine. The case presented here was an unusual, lifethreatening Pheochromocytoma. The selection of the appropriate treatment is critical to terminate the effects of catecholamine storm and to prepare for surgical resection of the tumor.

\section{References}

1. Olson SW, Deal LE, Piesman M (2004) Epinephrine- Secreting Pheochromocytoma presenting with Cardiogenic Shock and Profound Hypocalcemia. Ann Intern Med 140(10): 849-851.

2. Przemyslaw GuzikT, Andrzej Wykretowicz (2005) Adrenal pheochromocytoma associated with dramatic cyclic hemodynamic fluctuations. International Journal of Cardiology 103(3): 351-353.

3. Schurmeyer TH, Engeroff B, Dralle E, von zur Muhlen A (2005) Cardiological effects of catecholamine-secreting tumors. Eur J Clin Invest 27(3): 189-95.

4. Liao WB, Liu CF, Chiang CW, Kung CT, Lee CW (2000) Cardiovascular manifestations of pheochromocytoma. Am J Emerg Med 18(5): 622-25.

5. Gifford RW Jr, Manger WM, Bravo EL (1994) Pheochromocytoma. Endocrinol Metab Clin North Am 23: 387-404.

6. Cohn JN (1996) Paroxysmal hypertension and hypovolemia. N Engl J Med 275(12): 643-646.

7. Kobal SL, Paran E, Jamali A, Mizrahi S, Siegel RJ, et al. (2008) Pheochromocytoma: cyclic attacks of hypertension alternating with hypotension. Nat Clin Pract Cardiovasc Med 5(1):53-57.

8. Plouin PF, Duclos JM, Soppelsa F (2001) Factors associated with perioperative morbidity and mortality in patients with pheochromocytoma: analysis of 165 operations at a single center. J Clin Endocrinol Metab 86(4):1480-1486. 
ISSN: 2574-1241

DOI: $10.26717 /$ BJSTR.2020.25.004274

Liqun zhao. Biomed J Sci \& Tech Res

(c) This work is licensed under Creative

Submission Link: https://biomedres.us/submit-manuscript.php

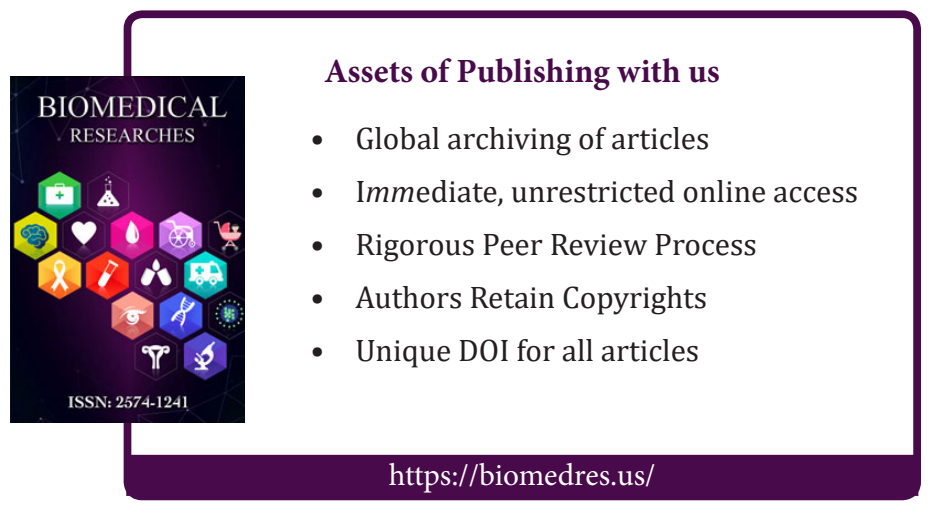

